\title{
Thermal, physical properties and flammability of silane treated kenaf/ pineapple leaf fibres phenolic hybrid composites
}

\begin{abstract}
Silane treated pineapple leaf fibre (PALF) and kenaf fibre were analyzed by Thermogravimetric analysis (TGA) that indicated the treated hybrid composite showed better thermal stability as compared to untreated hybrid composites. Dynamic mechanical analysis was carried out to evaluate the storage modulus $\left(E^{\prime}\right)$, loss modulus $\left(E^{\prime \prime}\right)$, and tan delta as a function of temperature. Storage modulus of treated hybrid composites displayed highest storage and loss modulus as in comparison of untreated hybrid composites. The peak heights of $\tan \alpha$ were highest in treated hybrid composites. Cole-Cole analysis was also carried out to understand the phase behaviour of the composite samples. Thermal mechanical analysis was used to study mechanical stability of hybrid composites in the presence of temperature. The effect of different fibre ratios in hybridization on density, void content, water absorption (WA), thickness swelling (TS) of PALF/KF hybrid composites were also analyzed. Treated hybrid composites were not very affective to improve the flammability of PALF/KF hybrid composites. The overall results showed that treated PALF/KF/phenolic hybrid composites improved the thermal and dynamic mechanical properties over untreated PALF/KF hybrid composites.
\end{abstract}

Keyword: Silane treatment; Thermo-gravimetric analysis; Dynamic mechanical analysis; Thermal mechanical analysis; Physical properties 